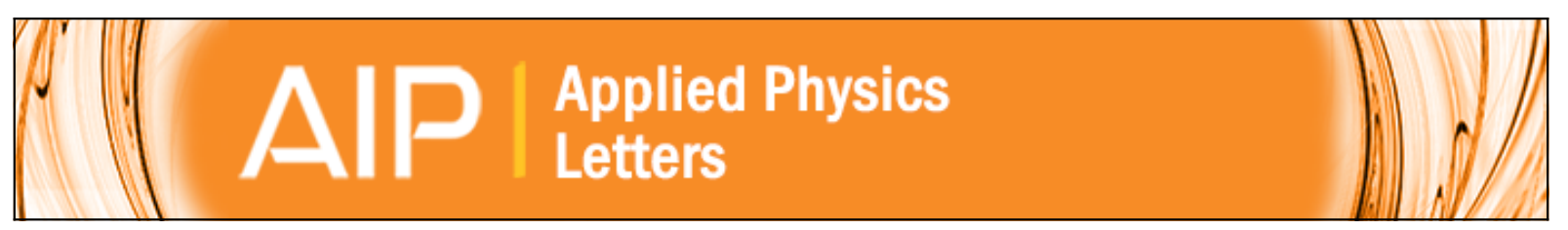

\title{
Bulk and surface sensitivity of a resonant waveguide grating imager
}

Norbert Orgovan, Boglarka Kovacs, Eniko Farkas, Bálint Szabó, Natalya Zaytseva, Ye Fang, and Robert Horvath

Citation: Applied Physics Letters 104, 083506 (2014); doi: 10.1063/1.4866460

View online: http://dx.doi.org/10.1063/1.4866460

View Table of Contents: http://scitation.aip.org/content/aip/journal/apl/104/8?ver=pdfcov

Published by the AIP Publishing 


\title{
Bulk and surface sensitivity of a resonant waveguide grating imager
}

\author{
Norbert Orgovan, ${ }^{1,2}$ Boglarka Kovacs, ${ }^{2}$ Eniko Farkas, ${ }^{2}$ Bálint Szabó, ${ }^{1}$ Natalya Zaytseva, ${ }^{3}$ \\ Ye Fang, ${ }^{3}$ and Robert Horvath ${ }^{2, a)}$ \\ ${ }^{1}$ Department of Biological Physics, Eötvös Loránd University, Pázmány P. stny. 1/A, H-1117 Budapest, \\ Hungary \\ ${ }^{2}$ Research Centre for Natural Sciences, Institute for Technical Physics and Materials Science, \\ Hungarian Academy of Sciences, Konkoly-Thege út 29-33, H-1120 Budapest, Hungary \\ ${ }^{3}$ Biochemical Technologies, Science and Technology Division, Corning Incorporated, Sullivan Park, \\ Corning, New York 14831, USA
}

(Received 25 September 2013; accepted 9 February 2014; published online 24 February 2014)

\begin{abstract}
We report the assessment of the sensitivity of a microplate-compatible resonant waveguide grating imager. The sensitivity to bulk refractive index changes was determined using a serial dilution of glycerol solution with the help of a refractometer. The surface sensitivity was examined using layer-by-layer polyelectrolyte films in conjunction with optical waveguide lightmode spectroscopy and characterized by the binding of acetazolamide to immobilized carbonic anhydrase under microfluidics. The results suggest that the imager has a limit of detection down to $2.2 \times 10^{-6}$ for refractive index change and $0.078 \mathrm{ng} / \mathrm{cm}^{2}$ for the adsorbed mass. (C) 2014 AIP Publishing LLC. [http://dx.doi.org/10.1063/1.4866460]
\end{abstract}

Label-free optical biosensors include surface plasmon resonance (SPR), ${ }^{1}$ optical waveguide lightmode spectroscopy (OWLS), ${ }^{2}$ photonic crystal (PC) biosensor, ${ }^{3}$ and resonant waveguide grating (RWG), ${ }^{4,5}$ all of which employ surface bound evanescent waves to characterize processes accompanied by refractive index variations close to the sensor surface $(100-200 \mathrm{~nm}){ }^{6}$ These biosensors, although divergent in throughput and operational schemes, have found widespread applications for both biomolecular interaction analysis $^{7,8}$ and cell phenotypic profiling. ${ }^{9-11}$ To overcome throughput limitation posed by SPR and OWLS, RWG employs nano-grating waveguide biosensors embedded in the Society for Biomolecular Screening compatible microplates, e.g., 96-well, 384-well, and 1536-well microplates. ${ }^{9,10}$ The RWG readers, in particular the recently developed imager, ${ }^{5,12}$ permit high-throughput and highly sensitive drug profiling and screening, and in-depth analysis of receptor signaling pathways in living cells. ${ }^{10}$ The RWG imager uses a tunable light source to interrogate simultaneously all biosensors in the microplate with a temporal resolution of $3 \mathrm{~s}$ and a spatial resolution of $\sim 80 \mu \mathrm{m} .{ }^{12}$ The illumination is achieved through a broadband light source in conjunction with a high precision narrow-band optical filter so it can sweep the wavelength from 823 to $838 \mathrm{~nm}$ in a stepwise fashion, each with $100 \mathrm{pm}$ every $20 \mathrm{~ms}$. This imager has a low thermal noise of $0.18 \pm 0.03 \mathrm{pm}(\mathrm{n}=384)$ for a whole plate under temperature controlled environment $\left(22^{\circ} \mathrm{C}\right)$, as obtained by monitoring the fluctuation of baseline signals. This imager is advantageous in that it allows online quality control and offline data filtration to improve assay quality. Given the importance to quantify biochemical interactions in vitro and in living cells and to compare performance among different biosensors, ${ }^{13}$ we herein set to determine the bulk and surface sensitivity of a RWG imager, Epic ${ }^{\circledR}$ BT system (Corning Incorporated, Corning, NY, USA).

\footnotetext{
a)E-mail: horvathr@mfa.kfki.hu
}

We first examined the sensitivity of the RWG imager to changes in bulk refractive index. The imager was first used to record the biosensor signals responding to a serial dilution of glycerol with water (VWR International, Debrecen, Hungary) in an Epic ${ }^{\circledR}$ 96-well uncoated microplate (Corning) under ambient temperature. Optical gratings at the bottom of each well enable incoupling of resonant light at a specific resonant wavelength $(\lambda)$ into the thin waveguide layer made of $\mathrm{Nb}_{2} \mathrm{O}_{5}$. The shifts in resonant wavelength of all sensors are detected with a precision of $0.25 \mathrm{pm}$ using a complementary metal-oxide semiconductor (CMOS) camera in conjunction with an online processor. Results showed that the biosensors in an uncoated 96-well plate gave rise to resonant peaks with full widths at half maximum of $1.593 \mathrm{~nm}$ and resonant wavelengths of $828.2 \mathrm{~nm}$, leading to a low quality factor of 520 . The glycerol dilution studies showed that the biosensor signals increased as the concentration of glycerol increased up to $50 \%$; however, the biosensor failed when the undiluted glycerol was examined (Fig. 1(a)). A Rudolph J157 table top refractometer (Rudolph Research Analytical, Hackettstwon, NJ, USA) was then used to determine in parallel the refractive index values of glycerol solutions. The refractometer has a precision of $10^{-5}$ refractive index units (RIU). Linear regression analysis revealed that the biosensor response, in terms of wavelength shift $\Delta \lambda$, was a linear function of the change in refractive index $(\Delta n)$ (Fig. 1(b)):

$$
\Delta \lambda=(-47 \pm 37) \mathrm{pm}+\Delta n(113850 \pm 1257) \mathrm{pm} .
$$

Using this equation, we estimated the detection range of the imager for bulk refractive index changes. With the bulk refractive index sensitivity of $113.85 \mathrm{~nm} / \mathrm{RIU}$ and the resolvability of $0.25 \mathrm{pm}$ wavelength shift of the imager, we estimated its lower limit of detection in $\Delta n\left(\Delta n_{\min }\right)$ to be 2.2 $\times 10^{-6}$ RIU. This is approximately one order of magnitude better than the bulk refractive index resolution of OWLS ${ }^{14-16}$ or a PC biosensor using plastic substrates $\left(3.4 \times 10^{-5} \mathrm{RIU}\right),{ }^{17}$ 
(a)

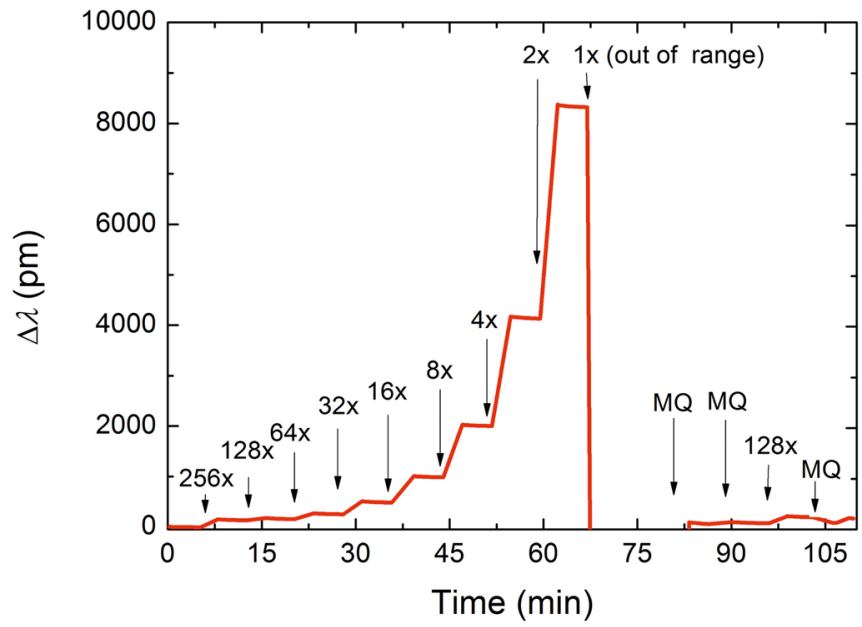

(b)

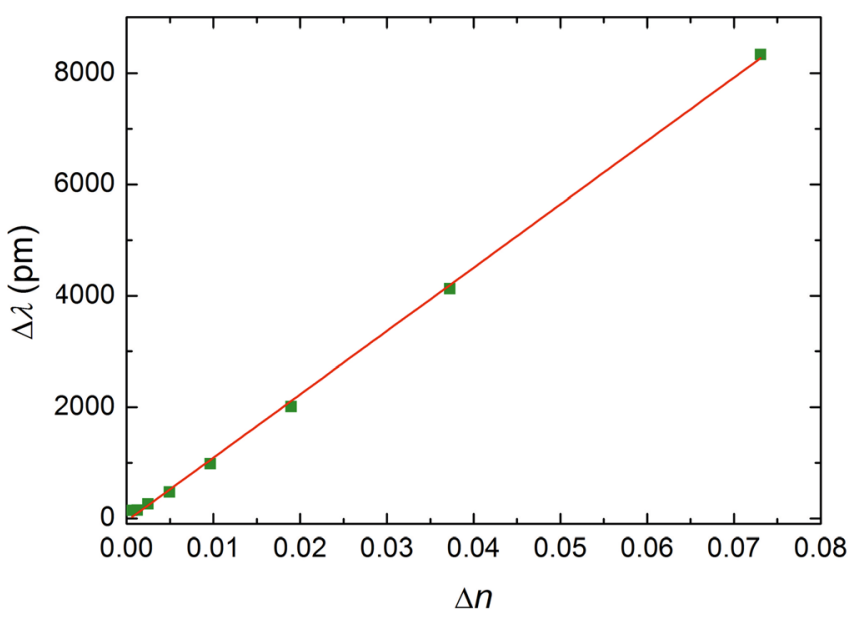

FIG. 1. The bulk refractive index sensitivity of the RWG imager. (a) Representative kinetic responses of a RWG biosensor to increasing concentrations of glycerol, recorded as the shifts in the resonant wavelength $(\Delta \lambda)$. The numbers indicate the fold of dilution. The baseline was established after the biosensor was equilibrated with Milli-Q water until the signal drifting became steady ( $<5$ pm within 5 min). (b) The resonant wavelength shift as a function of bulk refractive index (data shown as points, line is the fit to obtain the calibration equation).

but is largely within the range for SPR $\left(10^{-5}-10^{-8} \mathrm{RIU}\right) .^{13}$ However, the practical limit of detection for $\Delta n_{\text {min }}$ observed was $\sim 10^{-4}$ under the non-optimal experimental conditions used (i.e., manual washing, uncontrolled temperature, and the open environment). Of note, temperature fluctuations have an important effect on the limit of detection. It is important to stress that the refractive index of water shifts by approximately $8 \times 10^{-6}$, when its temperature is changed by $0.1^{\circ} \mathrm{C}$. $^{18}$ Therefore, limit of detection below $10^{-6}$ RIU requires mitigating temperature fluctuations or some kind of data averaging and filtration. Given that the imager sweeps the wavelength from 823 to $838 \mathrm{~nm}$, the maximum refractive index change $\left(\Delta n_{\max }\right)$ it can detect was estimated to be $\sim 0.13$. This suggests that the imager will fail to produce meaningful reading when $\Delta n>\Delta n_{\max }$, as evidenced by its failure to detect the real response to $100 \%$ glycerol (Fig. 1(a)). Of note, this detectable $\Delta n_{\max }$ is sufficient for almost all biological applications.

Next, we determined the surface sensitivity of the imager using two different approaches, layer-by-layer assembly of two distinct polyelectrolytes under ambient condition and direct binding of acetazolamide to immobilized carbonic anhydrase under temperature-controlled microfluidic system. The imager only measures the resonant wavelength of the zeroth order TM mode (but not that of the transverse electric (TE) mode) of the biosensor, preventing direct determination of the mass surface coverage of adsorbed species. Therefore, we used dual mode (TE and TM modes) OWLS as a complementary technique ${ }^{14-16}$ to determine both the thickness $d_{\mathrm{A}}$ and effective refractive index $n_{\mathrm{A}}$ of the adsorbed layer. The adsorbed mass density $M$ can then be obtained using the de Feijter formula ${ }^{19,20}$

$$
M=d_{\mathrm{A}} \frac{n_{\mathrm{A}}-n_{\mathrm{C}}}{\mathrm{d} n / \mathrm{d} c},
$$

where $n_{\mathrm{C}}, \quad c$, and $\mathrm{d} n / \mathrm{d} c$ are the refractive index, the concentration, and the refractive index increment of the solution containing the adsorbing species, respectively. Here, the only remaining unknown parameter, $\mathrm{d} n / \mathrm{d} c$, need to be determined using refractometry.

For layer-by-layer assembly studies under ambient condition, we used negatively charged poly(sodium 4-styrenesulfonate) (PSS) with an averaged molecular weight of $\sim 70 \mathrm{kDa}$ (Sigma-Aldrich Chemie GmbH, Munich, Germany) and positively charged poly(allylamine hydrochloride) (PAH) with an averaged molecular weight of $\sim 160 \mathrm{kDa}$ (Alfa Aesar GmbH, Karlsruhe, Germany). The refractive index values of the two polyelectrolytes were first determined using the refractometer. Results showed that the refractive index increment $\mathrm{d} n / \mathrm{d} c$ was $0.177 \pm 0.003 \mathrm{~cm}^{3} / \mathrm{g}$ for PSS and $0.214 \pm 0.003 \mathrm{~cm}^{3} / \mathrm{g}$ for PAH at room temperature. These values obtained were to large degree consistent with that of proteins reported in literature $\left(0.182 \mathrm{~cm}^{3} / \mathrm{g}\right) .^{21} \mathrm{We}$ then monitored the layer-by-layer assembly process of PAH and PSS deposited in an alternated manner using OWLS (OW2400 sensors, Microvacuum Ltd., Budapest, Hungary). Here, both PAH and PSS were dissolved in $10 \mathrm{mM} 4$-(2-hydroxyethyl)-1-piperazineethanesulfonic acid (HEPES)-KOH buffer, pH 7.4 to a final concentration of $1 \mathrm{mg} / \mathrm{ml}$. After establishing the baseline, the buffer was exchanged with the PAH solution. Adsorption was monitored until the signal became saturated. The solution was then removed and the OWLS cuvette was intensively rinsed with the buffer, and the biosensor signals were finally registered. The same steps were performed sequentially with PSS and then PAH solutions until five bilayers of PAH/PSS were deposited onto the sensor surface. All solutions injected into the OWLS cuvette equipped with a septum were $200 \mu \mathrm{l}$. Results showed that the layer-by-layer assembly process of PAH and PSS led to successive increase in the measured effective refractive indices, $N_{\mathrm{TM}}$ and $N_{\mathrm{TE}}$, corresponding to the zeroth order TM and TE polarized waveguide modes (Fig. 2(a)). Next, we monitored the layer-by-layer assembly of PAH and PSS up to five bilayers using the RWG imager. Here, solution amounts of $50 \mu \mathrm{l}$ were introduced into the wells of the Epic ${ }^{\circledR}$ microplate by manual pipetting. Results showed that the 
imager detected a sequentially increased signal as the PAH/PSS multilayer was built up (Fig. 2(b)). The total biosensor responses obtained using either OWLS or the RWG imager were found to be in linear relation with the numbers of bilayers deposited (Fig. 2(c)). These results confirm that the alternated deposition of PAH and PSS led to linearly growing polyelectrolyte layers. Finally, we estimated the surface sensitivity of the RWG imager through comparison with the OWLS data. Although OWLS and RWG use different waveguide films $\left(\mathrm{Si}_{0.25} \mathrm{Ti}_{0.75} \mathrm{O}_{2}{ }^{22}\right.$ versus $\mathrm{Nb}_{2} \mathrm{O}_{5}{ }^{4}$, respectively), this comparison is possible because the effect of the original sensor surface on adsorption is mostly masked by the first 1-2 layers of polyelectrolytes, ${ }^{23,24}$ so subsequently deposited layers can be considered to be similar and independent on the original biosensor surface. This was also supported by the overlapped results obtained using both systems (Fig. 2(c)). Therefore, we can calculate the surface mass density of the deposited polyelectrolyte multilayer from the OWLS data using Eq. (2) and the intermediate refractive index increment value $\left(0.1955 \mathrm{~cm}^{3} / \mathrm{g}\right)$ between PAH and PSS (Fig. 2(d)). A linear fit to the data resulted in

$$
\begin{aligned}
\Delta M= & (4.22 \pm 6.12) \mathrm{ng} / \mathrm{cm}^{2} \\
& +\Delta \lambda\left(3.1 \cdot 10^{-1} \pm 5 \cdot 10^{-3}\right) \mathrm{ng} / \mathrm{cm}^{2} / \mathrm{pm} .
\end{aligned}
$$

(a)

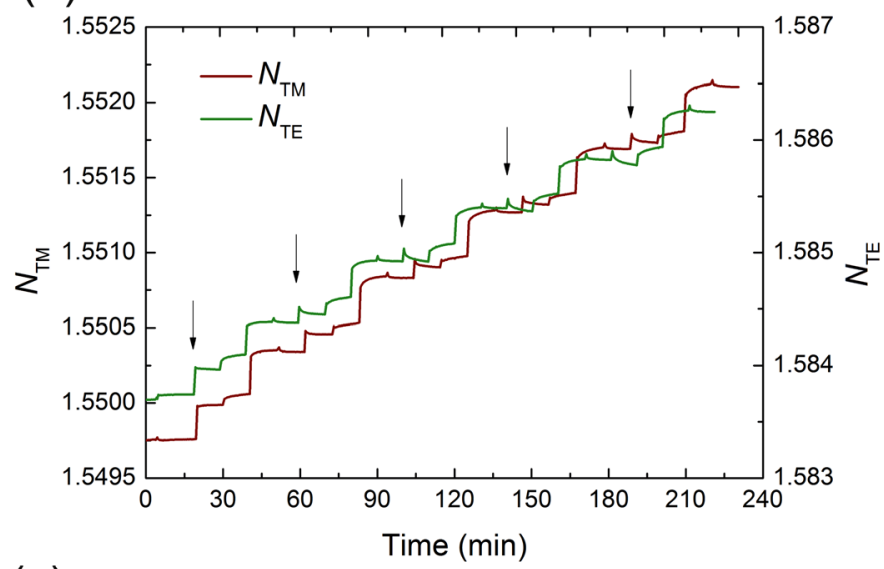

(c)

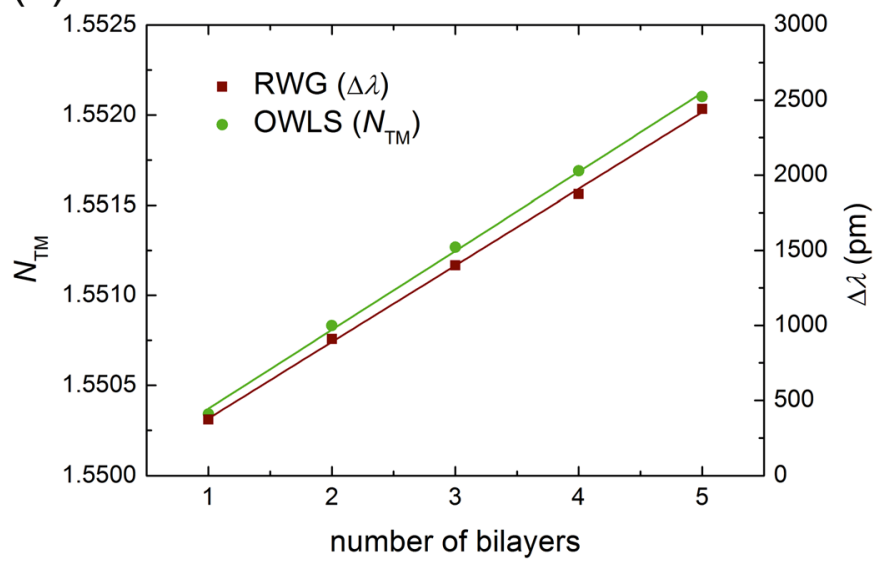

For the binding of small molecules to immobilized carbonic anhydrase II (CA) under temperature controlled microfluidic condition, we used a recently developed microfluidic RWG imager system. ${ }^{25}$ This system employs an onboard microfluidic system and the same RWG imager to measure the binding kinetics of small molecules to immobilized proteins in a 32-flow cell microchannel plate with intrawell selfreferencing. The immobilization of CA (Sigma-Aldrich, St. Louis, MO, USA) was achieved through perfusion of the biosensor having ethylene/maleic anhydride polymer coating with $50 \mu \mathrm{g} / \mathrm{ml} \mathrm{CA}$ at $\mathrm{pH} 5.4$ for $20 \mathrm{~min}$, followed by deactivating residual reactive groups on the surface with $0.2 \mathrm{M}$ ethanolamine- $\mathrm{HCl}(\mathrm{pH} 8.5)$ for $7 \mathrm{~min}$. After continuous perfusion with the binding buffer (3\% dimethyl sulfoxide in $10 \mathrm{mM} \mathrm{Na} 2 \mathrm{HPO}_{4}-\mathrm{KH}_{2} \mathrm{PO}_{4}, 137 \mathrm{mM} \mathrm{NaCl}, 2.7 \mathrm{mM} \mathrm{KCl}, \mathrm{pH}$ 7.4) for $3 \mathrm{~min}$, the total amount of immobilized proteins was determined and found to be $2150 \pm 66 \mathrm{pm}(\mathrm{n}=32)$. After immobilization, the baseline was reestablished, and the binding kinetics measurements were then performed by perfusion of the biosensors with a solution containing a small molecule of different doses, each in duplicate, for $1 \mathrm{~min}$, followed by the binding buffer for $\sim 3 \mathrm{~min}$, all at a flow rate of $100 \mu \mathrm{l} / \mathrm{min}$. Results showed that the sensorgrams for the binding of acetazolamide (molecular weight of $222 \mathrm{Da}$ ) were concentration dependent and saturable (Fig. 3). The biosensor response

(b)

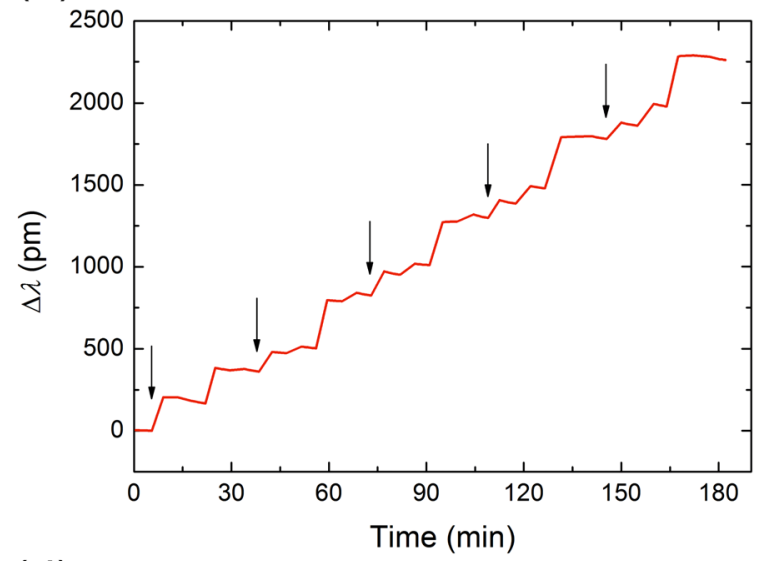

(d)

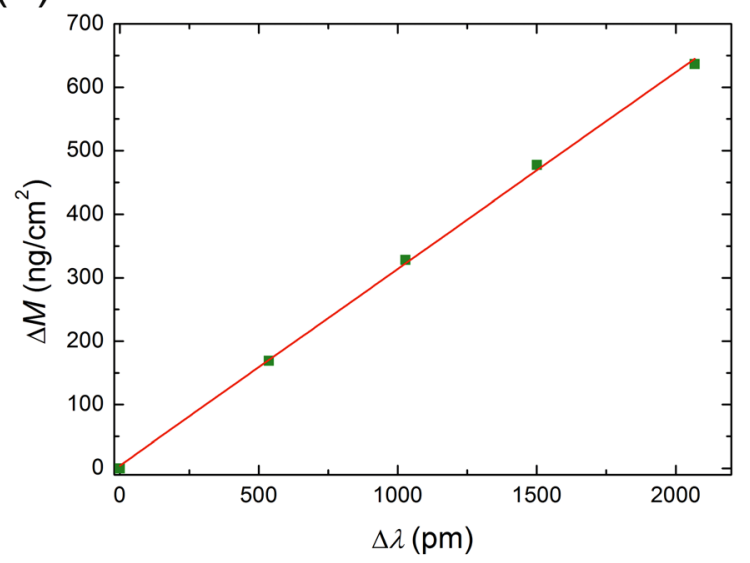

FIG. 2. The surface sensitivity of the RWG imager. (a) and (b) Representative signals arising from the layer by layer deposition of the (PAH-PSS) 5 polyelectrolyte multilayer as measured with OWLS (a), and the RWG imager (b). The arrows indicate the injections of the PAH solution. (c) The PAH-PSS polyelectrolyte multilayer grew linearly with the number of added bilayers as measured with OWLS (green dots) and with the RWG imager (red dots). The adjusted R-square values of the linear fits are $>0.998$ in both cases. (d) Surface mass density calculated from OWLS data is correlated with the response (data are shown as points) measured with the RWG imager to obtain a calibration equation (line). RWG data presented in (c) and (d) are a mean of responses measured in at least two wells and background corrected. Here, only the wavelength shifts relative to the 1st deposited bilayer were taken into account. 


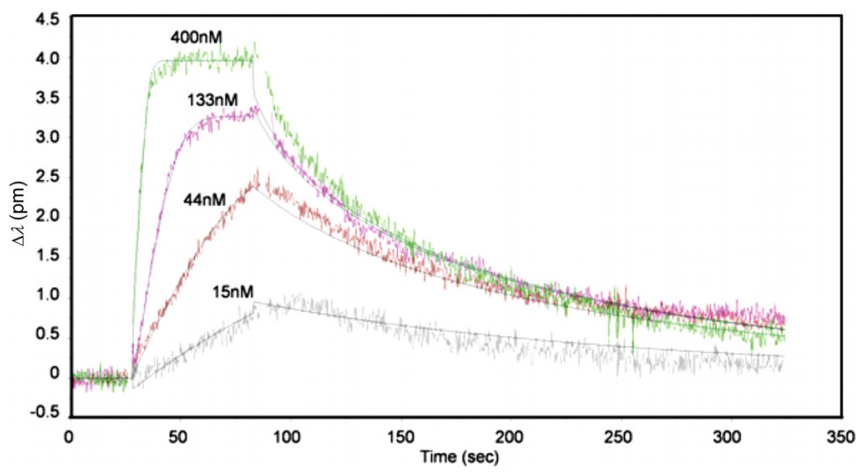

FIG. 3. The sensorgrams of acetazolamide binding to carbonic anhydrase II using microfluidic RWG imager system under temperature controlled environment.

increases during the association phase when acetazolamide binds to the immobilized proteins, while the response decreases during the dissociation phase when the buffer solution is introduced and acetazolamide dissociates from the surface bound proteins. The binding data were found to fit well with a 1:1 binding model with mass transport using Biacore BiaEvalution software, leading to an on-rate constant $\left(k_{o n}\right)$ of $2.08 \times 10^{6} \mathrm{M}^{-1} \mathrm{~s}^{-1}$, an off-rate constant $\left(k_{o f f}\right)$ of $0.026 \mathrm{~s}^{-1}$, and a dissociation constant $\left(K_{\mathrm{D}}\right)$ of $0.013 \mu \mathrm{M}$, which are consistent with literature values $\left(k_{\text {on }}: 3.0 \pm 2.1 \times 10^{6} \mathrm{M}^{-1} \mathrm{~s}^{-1}\right.$; $\left.k_{\text {off: }}: 0.079 \pm 0.031 \mathrm{~s}^{-1} ; K_{D}: 0.031 \pm 0.011 \mu \mathrm{M}\right) .{ }^{26}$ The binding data obtained confirm that under optimal condition the imager can resolve wavelength shifts down to $0.25 \mathrm{pm}$ with a low thermal noise, and the signal of $0.5 \mathrm{pm}$ can be readily detected. Using these results and Eq. (3), we estimate that the imager has a limit of detection of adsorbed mass down to $0.078 \mathrm{ng} / \mathrm{cm}^{2}$, which is approximately one order of magnitude better than OWLS $\left(\sim 1 \mathrm{ng} / \mathrm{cm}^{2}\right),{ }^{16}$ but similar to a PC biosensor fabricated on a glass substrate $\left(0.042 \mathrm{ng} / \mathrm{cm}^{-2}\right),{ }^{27}$ and SPR $\left(\sim 0.1 \mathrm{ng} / \mathrm{cm}^{-2}\right) .{ }^{13}$ Assuming that the average size of a protein is $\sim 50 \mathrm{~nm}^{2}$ and the surface coverage of immobilized and functional proteins is $50 \%$, the imager is estimated to be capable of detecting a small molecule of $100 \mathrm{Da}$ binding to $\sim 8 \%$ immobilized proteins under optimal conditions, suggesting that, similarly to SPR and PC biosensors, the imager can be applied for fragment screening under optimal conditions. ${ }^{28}$ Note that for cell-based assays, the superior detection limit is less important, given that living cells generally have slightly higher baseline fluctuation signal than in vitro binding assay conditions, but most biosensor signals arising from receptor signaling in living cells are greater than $30 \mathrm{pm}$.

In conclusion, we investigated the bulk and surface sensing capabilities of a RWG imager compatible to standard whole microplate. Parallel experiments with a table top refractometer and a single channel OWLS showed that the imager has a limit of detection down to $2.2 \times 10^{-6}$ for the bulk refractive index change, and $0.078 \mathrm{ng} / \mathrm{cm}^{2}$ for the adsorbed mass change. For RWG, further improvement can be achieved using imagers having higher spatial resolution so data averaging and filtration can be more effective, ${ }^{29}$ using on chip integrated biosensor systems, ${ }^{30,31}$ or using biosensors with high quality factors. ${ }^{32}$ Nonetheless, the present study establishes the calibration curve between the RWG biosensor signal and the adsorbed surface mass, allowing direct quantification of tiny variations in surface mass arising from the binding of biomolecules, or of dynamic mass redistribution arising from the activation of cell signaling in living cells using the highly sensitive RWG imager.

The present work was supported by the "Lendület" Program of the Hungarian Academy of Sciences.

${ }^{1}$ J. Homola, S. S. Yee, and G. Gauglitz, Sens. Actuators B Chem. 54, 3 (1999).

${ }^{2}$ J. Vörös, J. J. Ramsden, G. Csúcs, I. Szendro", S. M. De Paul, M. Textor, and N. D. Spencer, Biomaterials 23, 3699 (2002).

${ }^{3}$ B. T. Cunningham, P. Li, S. Schulz, B. Lin, C. Baird, J. Gerstenmaier, C. Genick, F. Wang, E. Fine, L. Laing, and B. O. Lin, J. Biomol. Screen. 9, 481 (2004).

${ }^{4}$ Y. Fang, A. M. Ferrie, N. H. Fontaine, J. Mauro, and J. Balakrishnan, Biophys. J. 91, 1925 (2006).

${ }^{5}$ N. Orgovan, B. Peter, Sz. Bősze, J. J. Ramsden, B. Szabó, and R. Horváth, Sci. Rep. 4, 4034 (2014).

${ }^{6}$ K. Tiefenthaler and W. Lukosz, J. Opt. Soc. Am. B 6, 209 (1989).

${ }^{7}$ Y. Fang, Expert Opin. Drug Discov. 7, 969 (2012).

${ }^{8}$ S. Pal, P. M. Fauchet, and B. L. Miller, Anal. Chem. 84, 8900 (2012).

${ }^{9}$ Y. Fang, Int. J. Electrochem. 2011, 460850 (2011).

${ }^{10}$ Y. Fang, Expert Opin. Drug Discov. 6, 1285 (2011).

${ }^{11}$ S. M. Shamah and B. T. Cunningham, Analyst 136, 1090 (2011).

${ }^{12}$ A. M. Ferrie, Q. Wu, and Y. Fang, Appl. Phys. Lett. 97, 223704 (2010).

${ }^{13}$ X. Fan, I. M. White, S. I. Shopova, H. Zhu, J. D. Suter, and Y. Sun, Anal. Chim. Acta 620, 8 (2008).

${ }^{14}$ R. Horvath, J. McColl, G. E. Yakubov, and J. J. Ramsden, J. Chem. Phys. 129, 071102 (2008).

${ }^{15}$ N. Kovács, D. Patkó, N. Orgován, S. Kurunczi, J. J. Ramsden, F. Vonderviszt, and R. Horvath, Anal. Chem. 85, 5382 (2013).

${ }^{16}$ S. Kurunczi, A. Hainard, K. Juhasz, D. Patko, N. Orgovan, N. Turck, J. C. Sanchez, and R. Horvath, Sens. Actuators B 181, 71 (2013).

${ }^{17}$ B. Cunningham, P. Li, B. Lin, and J. Pepper, Sens. Actuators B 81, 316 (2002).

${ }^{18}$ G. Abbate, U. Bernini, E. Ragozzino, and F. Somma, J. Phys. D: Appl. Phys. 11, 1167 (1978).

${ }^{19}$ J. J. Ramsden, J. Stat. Phys. 73, 853 (1993).

${ }^{20}$ J. A. de Feijter, J. Benjamins, and V. A. Veer, Biopolymers 17, 1759 (1978).

${ }^{21}$ R. Barer and S. Joseph, Q. J. Microsc. Sci. 95, 399 (1954).

${ }^{22}$ R. Horvath, L. R. Lindvold, and N. B. Larsen, Appl. Phys. B 74, 383 (2002).

${ }^{23}$ G. Decher, Science 277, 1232 (1997).

${ }^{24}$ J. J. Ramsden, Yu. M. Lvov, and G. Decher, Thin Solid Films 254, 246 (1995).

${ }^{25}$ N. Zaytseva, W. Miller, V. Goral, J. Hepburn, and Y. Fang, Appl. Phys. Lett. 98, 163703 (2011).

${ }^{26}$ G. A. Papalia, S. Leavitt, M. A. Bynum, P. S. Katsamba, R. Wilton, H. Qiu, M. Steukers, S. Wang, L. Bindu, S. Phogat et al., Anal. Biochem. 359, 94 (2006).

${ }^{27}$ B. Cunningham, B. Lin, J. Qiu, P. Li, J. Pepper, and B. Hugh, Sens. Actuators B 85, 219 (2002).

${ }^{28}$ S. Geschwindner, N. Dekker, R. Horsefield, A. Tigerström, P. Johansson, C. W. Scott, and J. S. Albert, J. Med. Chem. 56, 3228 (2013).

${ }^{29}$ A. M. Ferrie, O. D. Deichmann, Q. Wu, and Y. Fang, Appl. Phys. Lett. 100, 223701 (2012).

${ }^{30}$ C. J. Choi, A. R. Belobraydich, L. L. Chan, P. C. Mathias, and B. T. Cunningham, Anal. Biochem. 405, 1 (2010).

${ }^{31}$ D. Threm, Y. Nazirizadeh, and M. Gerken, J. Biophotonics 5, 601 (2012).

${ }^{32}$ F. Vollmer and S. Arnold, Nat. Methods 5, 591 (2008). 